\title{
The phosphodiesterase 5 inhibitor, sildenafil, further augments the compensatory upregulation of NO-cGMP signalling in hypoxic pulmonary hypertension
}

\author{
Mark Kirsch*1, Norbert Weissmann², Barbara Kemp-Harper, \\ Friedrich Grimminger ${ }^{2}$ and Harald HHW Schmidt ${ }^{1,3}$
}

\author{
Address: ${ }^{1}$ Rudolf-Buchheim-Institut für Pharmakologie, Justus-Liebig-Universität, Gießen, Germany, 2Zentrum für Innere Medizin, Justus-Liebig- \\ Universität, Gießen, Germany and ${ }^{3}$ Monash University, Dept. of Pharmacology, Melbourne, Australia \\ Email: Mark Kirsch* - mark.kirsch@tiscali.de \\ * Corresponding author
}

from 2nd International Conference of cGMP Generators, Effectors and Therapeutic Implications

Potsdam, Germany, 10-12 June, 2005

Published: 16 June 2005

BMC Pharmacology 2005, 5(Suppl I):P26 doi:I0.II86/I47I-22I0-5-SI-P26

Hypoxic pulmonary hypertension is a severe syndrome with a complex underlying pathophysiology. The mechanisms which lead to hypoxic pulmonary hypertension $(\mathrm{HPH})$ remain incompletely understood and an impairment of the vaso- and bronchodilatory NO-cGMP signalling cascade has been suggested. Clinically, the specific phosphodiesterase 5 inhibitor sildenafil reduces pulmonary remodeling and hypoxic pulmonary resistance, but it is unclear whether this effect is mechanisms-based, ie PDE5 is involved in the pathophysiology of HPH. Here we report changes in protein expression and activity of all key enzymes of the NO-cGMP signalling cascade in lungs of mice kept under normobaric hypoxia $\left(10 \% \mathrm{O}_{2}\right)$ for 5 and 22 days versus normobaric controls. We also examined the effects of acute sildenafil treatment in isolated perfused lungs following exposure to hypoxia for $5 \mathrm{~d}$ and the possible role of the the counter player of $\mathrm{NO}$, the superoxide generating NADPH oxidase (Nox) 4. Superoxide can scavenge NO to yield peroxynitrite which in turn nitrates protein tyrosin residues; immunopositive nitrotyrosine of cellular proteins was thus taken as a marker of oxidative/nitrative stress. Phosphorylation of the cGK substrate, vasodilator stimulated phosphoprotein (VASP), was taken as an overall marker for the effectiveness of NOcGMP signalling. Surprisingly, NOS III and NOS II expression were significantly increased as early as 5 days of hypoxia $(192 \pm 16 \%$ and $222 \pm 34 \%$ of control, $\mathrm{p}<0.01)$ and remained elevated after $22 \mathrm{~d}$ of hypoxia $(179 \pm 10 \%$ and $180 \pm 7 \%$ of control, $\mathrm{p}<0.01$ ). Consistently, cGMP accumulation in the perfusate of isolated lungs from mice exposed 5d to hypoxia was significantly increased as com- pared with normoxic lungs. In contrast, to recent studies, we found unchanged protein expression of sGC $\alpha_{1} / \beta_{1}$ and cGMP dependent phosphodiesterase as well as sustained basal and DEA-NO $(300 \mu \mathrm{M})$ stimulated in vitro sGC $\alpha_{1} /$ $\beta_{1}$ activity and cGMP-dependent phosphodiesterase activity. However, protein expression of cGMP-dependent protein kinase (cGKI) was significantly decreased after $22 \mathrm{~d}$ of hypoxia $(83 \pm 5.3 \%$ of control, $\mathrm{p}<0.01)$. The net result of these counterveiling effects on cGMP signalling as evidenced by VASP phosphorylation were surprisingly increased after 22 days of hypoxia $(350 \pm 84.5 \%$ of norm, $\mathrm{p}<0.01$ ). Immunopositive tyrosine nitration of cellular proteins after 5 and $22 \mathrm{~d}$ of hypoxia were unchanged suggesting that the bioavailability of NO was not affected by reactive oxygen species and peroxynitrite formation. Also Nox 4 expression was unchanged. Taken together, our results suggest that NO-cGMP signalling is not primarily affected in HPH but rather upregulated in a compensatory fashion; thus further inhibition of cGMP degradation by sildenafil do not target a pathomechanism per se but enhance these auto-protective mechanisms in the setting of $\mathrm{HPH}$. 